\title{
Gravitational Radiation from Keplerian Binary with Oscillating Orbital Plane
}

\author{
S. B. Faruque ${ }^{1}$, M. A. Nawaz, and M. H. Ahsan \\ Department of Physics, Shahjalal University of Science and Technology, Sylhet 3114, Bangladesh
}

Received 7 September 2008, in final revised form 8 December 2008

\begin{abstract}
The gravitational radiation from two point masses in Keplerian circular or elliptic orbit is calculated with the assumption that the orbital plane of the binary undergoes small oscillation about the equilibrium $\mathrm{x}-\mathrm{y}$ plane. This problem is simplification of a physically possible orbit where one of the point masses is spinning whereby the spin-orbit force drives the orbital plane to wobble in a complicated manner. It is shown that the total energy of gravitational radiation emitted by the binary in this case is greater than the energy emitted when the binary orbit is fixed in the $x-y$ plane. The results presented are in fact a generalization of the classic results of Landau and Lifshitz.
\end{abstract}

Keywords: Gravitational wave; Energy; Binary; Oscillating orbit.

(c) 2009 JSR Publications. ISSN: 2070-0237(Print); 2070-0245 (Online). All rights reserved.

DOI: 10.3329/jsr.vlil.1093

\section{Introduction}

We are now passing the exciting days of gravitational wave (GW) research. Earth-based laser-interferometric detectors of gravitational wave are now collecting data, and LIGO has just completed the longest scientific run [1] to date. The space-based detector LISA is expected to open an observational window at low frequencies within the next decade [2]. In such an exciting and important time of gravitational wave research, every bit of information on gravitational radiation, be it theoretical, computational or experimental, is valuable. The most basic of GW science is the energy of radiation emitted by a GW source such as a binary star, a binary black hole etc. Calculation of gravitational radiation energy emitted by point masses in Keplerian elliptical orbit was performed by Peters and Mathews [3], by Landau and Lifshitz [4]. In current literature, the objectives of gravitational wave research are more focused on detection of GW [5-8] and on evolution of GW sources [9-12]. In the present paper, we revisit the GW energy emission problem with a fresh look in the scenario. In most astrophysical binaries, the orbital plane does not remain invariant on a plane. For example, in a binary where one of the bodies is spinning, the spin-orbit force drives the orbital plane to precession [13] or to oscillation in a

\footnotetext{
${ }^{1}$ Corresponding author: awsbf62@yahoo.com
} 
complex manner [14]. Such precession or oscillation modulates the GW signal and the total energy emitted also changes. Here we consider a simplified problem from the scenario reported in [14]. Let two point masses in a Keplerian binary revolve round the center-of-mass in circular orbit and at the same time, the plane of the orbit is undergoing small oscillation about the equilibrium $x-y$ plane. We assume that the angular frequency of oscillation about the $x-y$ plane is equal to the Keplerian angular frequency of orbital motion in the $x-y$ plane. Moreover, we assume that the amplitude of angular oscillation about the $x-y$ plane is very small compared to the radius of the orbit. We then calculate the energy emitted separately in the two polarization modes of gravitational wave and the total energy emitted in all directions. We find that the amount of energy emitted gets increased which is determined by the amplitude of angular oscillation. This is an important finding, and we feel that many researchers would like to know the way to this result. The paper is organized as follows: In section 2, we briefly summarize the important formulae of gravitational wave. In section 3, we present the problem and the subsequent calculation. Finally, conclusion is presented in section 4 .

\section{Gravitational Radiation}

Gravitational radiation emission from various astrophysical sources has been the focus of many researches (see, for example, refs. [15-21]). Let us consider a source of gravitational radiation characterized by a mass quadrupole moment tensor $D_{\alpha \beta}$ with the six elements $D_{x x}, D_{y y}, D_{z z}, D_{x y}, D_{y z}, D_{z x}$, with respect to a set of fixed inertial axes (x,y,z). We define $D_{\alpha \beta}$ as in [4], that is,

$$
D_{\alpha \beta}=\int \rho\left(3 x^{\alpha} x^{\beta}-\delta_{\alpha \beta} r^{2}\right) d V
$$

where $\rho$ is the mass density, and $r^{2}=x^{2}+y^{2}+z^{2}, d V=d x d y d z$. The waves can be taken to be plane in view of the typically large distance between the source and the observer. The two independent polarization states of the gravitational wave can be represented by the three-dimensional symmetric, unit polarization tensor $e_{\alpha \beta}$ satisfying the relations

$$
e_{\alpha \alpha}=0, \quad e_{\alpha \beta} n_{\beta}=0, \quad e_{\alpha \beta} e_{\alpha \beta}=1,
$$

where $\hat{n}$ is a unit vector in the direction of propagation of the wave. Let us label the two polarizations by [3]

$$
e_{+}=\frac{1}{\sqrt{2}}(\hat{\theta} \hat{\theta}-\hat{\phi} \hat{\phi}), \quad e_{\times}=\frac{1}{\sqrt{2}}(\hat{\theta} \hat{\phi}+\hat{\phi} \hat{\theta}),
$$

where $\theta$ and $\phi$ are conventional polar coordinates. In this basis, the wave form can be written as [22]

$$
r h=\left(\ddot{D}_{\theta \theta}-\ddot{D}_{\phi \phi}\right) e_{+}+2 \ddot{D}_{\theta \phi} e_{\times},
$$


where $h$ is the metric perturbation or the GW wave form, and $D_{\theta \theta}, D_{\theta \phi}$ and $D_{\phi \phi}$ are the physical components of $D_{i j}$ (the Cartesian components of quadrupole tensor) projected along the directions of the spherical unit vectors $\hat{\theta}$ and $\hat{\phi}$. There exists canonical procedure for obtaining these components, but we simply quote the results from Ref. [22]:

$$
\begin{aligned}
& D_{\theta \theta}=\left(D_{x x} \cos ^{2} \phi+D_{y y} \sin ^{2} \phi+D_{x y} \sin 2 \phi\right) \cos ^{2} \theta+ \\
& +D_{z z} \sin ^{2} \theta-\left(D_{x z} \cos \phi+D_{y z} \sin \phi\right) \sin 2 \theta, \\
& D_{\phi \phi}=D_{x x} \sin ^{2} \phi+D_{y y} \cos ^{2} \phi-D_{x y} \sin 2 \phi, \\
& D_{\theta \phi}=-\frac{1}{2}\left(D_{x x}-D_{y y}\right) \cos \theta \sin 2 \phi+D_{x y} \cos \theta \cos 2 \phi+ \\
& +\left(D_{x z} \sin \phi-D_{y z} \cos \phi\right) \sin \theta .
\end{aligned}
$$

The expressions for the intensity of radiation of a given polarization into solid angle $d \Omega$ are [4]

$$
d I=\frac{G}{72 \pi c^{5}}\left(\frac{d^{3} D_{\alpha \beta}}{d t^{3}} e_{\alpha \beta}\right)^{2} d \Omega,
$$

where $G$ is the Newton's gravitational constant and $c$ is the speed of light in free space. Using Eqs. (3) and (4), we can write for the intensity of GW in $(\times)$ polarization as

$$
\frac{d I_{1}}{d \Omega}=\frac{G}{72 \pi c^{5}}\left(2 \frac{d^{3} D_{\theta \phi}}{d t^{3}} \frac{1}{\sqrt{2}}\right)^{2}=\frac{G}{36 \pi c^{5}}\left(\frac{d^{3} D_{\theta \phi}}{d t^{3}}\right)^{2},
$$

and that in $(+)$ polarization as

$$
\frac{d I_{2}}{d \Omega}=\frac{G}{72 \pi c^{5}}\left[\left(\frac{d^{3} D_{\theta \theta}}{d t^{3}}-\frac{d^{3} D_{\phi \phi}}{d t^{3}}\right) \frac{1}{\sqrt{2}}\right]^{2}=\frac{G}{144 \pi c^{5}}\left(\frac{d^{3} D_{\theta \theta}}{d t^{3}}-\frac{d^{3} D_{\phi \phi}}{d t^{3}}\right)^{2} .
$$

In the next section we apply these formulae to find out the gravitational radiation energy emitted by a Keplerian binary whose orbital plane is oscillating about the equilibrium x-y plane.

\section{Gravitational Radiation from a Binary with Oscillating Orbital Plane}

In many astrophysical binary star systems, the orbit of the stars undergoes precession and oscillation due to many perturbing forces, such as, spin-orbit, spin-spin interactions. Specifically, the spin-orbit force drives the orbital plane to oscillation about the equilibrium plane in a quite complicated manner. One typical case is analyzed in Ref. [14].

We consider a simplified situation defined by an almost fixed orbital plane confined to the $\mathrm{x}-\mathrm{y}$ plane, but the orbital plane undergoes very small angular oscillation about the 
equilibrium $x-y$ plane. This situation simulates some of the characteristics of orbital motion of a Keplerian binary with one particle having small spin. Now, we define the orbit by the following orbital variables:

$$
r=\text { constant, } \quad \theta_{0}=\frac{\pi}{2}-b \sin \omega t, \quad \phi_{0}=\omega t,
$$

where $\omega$ is the Newtonian angular frequency of the orbit in the x-y plane , $r=\left|\vec{r}_{1}-\vec{r}_{2}\right|$, $\vec{r}_{1}, \vec{r}_{2}$ being the positions of the particles of mass $m_{1}, m_{2}$, respectively, and $b$ is a very small parameter $(b<<1$ ) characterizing the angular oscillation about the $\mathrm{x}-\mathrm{y}$ plane. This oscillation is of the same frequency as the Newtonian orbital frequency. This choice is not whimsical, but can be considered as practical (see, for instance, Ref. [14]). Now, we can approximate the Cartesian components of the vector $\vec{r}$ as

$$
\begin{aligned}
& x \cong r \cos \omega t, \\
& y \cong r \sin \omega t, \\
& z \cong r b \sin \omega t .
\end{aligned}
$$

Then, the quadrupole moments are

$$
\begin{aligned}
& D_{x x}=\mu r^{2}\left(3 \cos ^{2} \omega t-1\right), D_{y y}=\mu r^{2}\left(3 \sin ^{2} \omega t-1\right), \\
& D_{x y}=\frac{3}{2} \mu r^{2} \sin 2 \omega t, D_{z z}=\mu r^{2}\left(3 b^{2} \sin ^{2} \omega t-1\right), \\
& D_{x z}=\frac{3}{2} \mu b r^{2} \sin 2 \omega t, D_{y z}=3 \mu b r^{2} \sin ^{2} \omega t,
\end{aligned}
$$

where $\mu=\frac{m_{1} m_{2}}{m_{1}+m_{2}}$, is the reduced mass of the binary. Now, we label the third derivatives of the D's as follows:

$$
\dddot{D}_{x x}=A, \dddot{D}_{y y}=B, \dddot{D}_{x y}=C, \dddot{D}_{z z}=D, \dddot{D}_{x z}=E, \dddot{D}_{y z}=F \text {. }
$$

Now, using Eqs. (5), (7), and (12), we obtain

$$
\begin{aligned}
& \frac{d I_{1}}{d \Omega}=\frac{G}{36 \pi c^{5}}\left(\frac{1}{2}\left[\frac{1}{4}\left\{4 C^{2}+(A-B)^{2}\right\} \cos ^{2} \theta+\frac{1}{4}\left\{4 C^{2}-(A-B)^{2}\right\} \cos ^{2} \theta \cos 4 \phi\right]\right. \\
& +\left(E^{2} \sin ^{2} \phi+F^{2} \cos ^{2} \phi\right) \sin ^{2} \theta-E F \sin ^{2} \theta \sin 2 \phi+\frac{1}{2} C(B-A) \cos ^{2} \theta \sin 4 \phi \\
& +\left\{\frac{1}{4} E(A-B)-\frac{1}{2} C F\right\} \sin 2 \theta \cos 3 \phi+\left\{\frac{1}{4} F(A-B)+\frac{1}{2} C E\right\} \sin 2 \theta \sin 3 \phi \\
& \left.+\left\{\frac{1}{4} F(A-B)-\frac{1}{2} C E\right\} \sin 2 \theta \sin \phi+\left\{\frac{1}{4} E(B-A)-\frac{1}{2} C F\right\} \sin 2 \theta \cos \phi\right)
\end{aligned}
$$

Since, the system is rapidly rotating about the z-axis, average over the angle $\phi$ is appropriate. Hence, we obtain 


$$
\frac{d I_{1}}{d \Omega}=\frac{G}{36 \pi c^{5}}\left[\frac{1}{2}\left\{C^{2}+\frac{1}{4}(A-B)^{2}\right\} \cos ^{2} \theta+\frac{1}{2}\left(E^{2}+F^{2}\right) \sin ^{2} \theta\right] .
$$

Next, we take an average over the orbital period and after a somewhat lengthy calculation we obtain the average

$$
\left\langle\frac{d I_{1}}{d \Omega}\right\rangle=\frac{G \mu^{2} r^{4} \omega^{6}}{2 \pi c^{5}}\left\{4\left(1-b^{2}\right) \cos ^{2} \theta+4 b^{2}\right\} .
$$

This equation gives the average intensity of gravitational radiation per unit solid angle which is dependent on the oscillation parameter $b$. Moreover, the result correctly reduces to the classic result of Landau and Lifshitz [4] for circular orbit with invariant orbital plane, i.e. when we put $b=0$. Now, the intensity of $(+)$ polarization is

$$
\frac{d I_{2}}{d \Omega}=\frac{G}{144 \pi c^{5}}\left[\begin{array}{l}
\left(A \cos ^{2} \phi+B \sin ^{2} \phi+C \sin 2 \phi\right) \cos ^{2} \theta+ \\
D \sin ^{2} \theta-(E \cos \phi+F \sin \phi) \sin 2 \theta \\
-\left(A \sin ^{2} \phi+B \cos ^{2} \phi-C \sin 2 \phi\right)
\end{array}\right]^{2}
$$

Averaging over the angle $\phi$ gives

$$
\frac{d I_{2}}{d \Omega}=\frac{G}{144 \pi c^{5}}\left[\begin{array}{l}
\cos ^{4} \theta\left\{\frac{3}{8}\left(A^{2}+B^{2}\right)+\frac{1}{2} C^{2}+\frac{1}{4} A B+D^{2}-2\left(E^{2}+F^{2}\right)-(A D+B D)\right\}+ \\
+\cos ^{2} \theta\left\{C^{2}-\frac{1}{4}\left(A^{2}+B^{2}\right)-\frac{3}{2} A B-2 D^{2}+2(A D+B D)+2\left(E^{2}+F^{2}\right)\right\}+ \\
+\frac{3}{8}\left(A^{2}+B^{2}\right)+\frac{1}{2} C^{2}+\frac{1}{4} A B+D^{2}-A D-B D
\end{array}\right]
$$

Next, averaging over the orbital period gives

$$
\left\langle\frac{d I_{2}}{d \Omega}\right\rangle=\frac{G \mu^{2} r^{4} \omega^{6}}{2 \pi c^{5}}\left[1+b^{4}+2\left(1+2 b^{2}-b^{4}\right) \cos ^{2} \theta+\left(1-4 b^{2}+b^{4}\right) \cos ^{4} \theta\right]
$$

This result also reduces to the classic result of Landau and Lifshitz [4] in the $b=0$ limit. The total intensity of GW radiation in the present context is found by summing Eq. (15) and (18). We obtain the total intensity as

$$
\left\langle\frac{d I}{d \Omega}\right\rangle=\frac{G \mu^{2} r^{4} \omega^{6}}{2 \pi c^{5}}\left[1+4 b^{2}+b^{4}+\left(6-2 b^{4}\right) \cos ^{2} \theta+\left(1-4 b^{2}+b^{4}\right) \cos ^{4} \theta\right]
$$

Integrating this expression over all directions, we get the energy radiated in gravitational wave in all directions per unit time as 


$$
-\frac{d E}{d t}=I=\frac{32 G \mu^{2} r^{4} \omega^{6}}{5 c^{5}}\left[1+b^{2}+\frac{b^{4}}{8}\right],
$$

which reduces to the classic result of Landau and Lifshitz [4], for the case of circular binary orbit fixed in the $\mathrm{x}-\mathrm{y}$ plane, using $b=0$, to

$$
I=\frac{32 G \mu^{2} r^{4} \omega^{6}}{5 c^{5}} .
$$

Comparing Eq. (20) with Eq. (21), we see that the total energy radiated in gravitational wave by a Keplerian binary system which is undergoing small oscillation of the orbital plane is more than that emitted by a binary system with invariant orbital plane. Hence, we found an important result, namely, the quantity of GW radiation emitted by an astrophysical binary that is undergoing small orbital plane oscillation about the equilibrium $x-y$ plane with angular amplitude $b$, given by Eq. (20). If $b \approx 0.1$, compatible with the approximation used in this work, then radiated GW energy increases by about $1 \%$. So, the contribution of the small oscillation considered here in this work is appreciable.

We now extend the calculation of the GW radiation to the case of elliptic binary orbit. In reality, astrophysical binary orbits are elliptic. The orbit may also undergo oscillation. Therefore, a calculation of the GW radiation output from such binaries will be worthwhile to be carried out. With this motivation, we proceed with a simplifying assumption that the orbital oscillation is of the same frequency as that of orbital revolution. The same formulae (7), (8), (14) and (17) give the gravitational radiation intensity in the present case. We define the orbital elements as that of a Keplerian elliptic orbit. In particular, we assume the following orbital parameters:

$$
\begin{aligned}
r & =\frac{a\left(1-e^{2}\right)}{1+e \cos \varphi}, \quad \theta=\frac{\pi}{2}-b \sin \varphi \\
\frac{d \varphi}{d t} & =\frac{\left[G\left(m_{1}+m_{2}\right) a\left(1-e^{2}\right)\right]^{1 / 2}}{r^{2}}
\end{aligned}
$$

where $a$ and e are the semi-major axis and eccentricity, respectively of an elliptic binary orbit. The quadrupole moments are

$$
\begin{aligned}
& D_{x x}=\mu r^{2}\left(3 \cos ^{2} \varphi-1\right), D_{y y}=\mu r^{2}\left(3 \sin ^{2} \varphi-1\right) \\
& D_{z z}=\mu r^{2}\left(3 b^{2} \sin ^{2} \varphi-1\right), D_{x y}=\frac{3}{2} \mu r^{2} \sin 2 \varphi \\
& D_{x z}=\frac{3}{2} \mu b r^{2} \sin 2 \varphi, D_{y z}=3 \mu b r^{2} \sin ^{2} \varphi .
\end{aligned}
$$

We keep the same notation as (12) for the third derivatives of the different quadrupole moments. Explicitly these derivatives are 


$$
\begin{aligned}
& A=\beta(1+e \cos \varphi)^{2}\left[6 \sin 2 \varphi+e \sin \varphi\left(9 \cos ^{2} \varphi-1\right)\right] \\
& B=-\beta(1+e \cos \varphi)^{2}\left[6 \sin 2 \varphi+e \sin \varphi\left(4+9 \cos ^{2} \varphi\right)\right] \\
& C=-\beta(1+e \cos \varphi)^{2}\left[6 \cos 2 \varphi-3 e \cos \varphi\left(1-3 \cos ^{2} \varphi\right)\right] \\
& D=-\beta(1+e \cos \varphi)^{2}\left[6 b^{2} \sin 2 \varphi+3 b^{2} e \sin \varphi\left(1+3 \cos ^{2} \varphi\right)+e \sin \varphi\right] \\
& E=-3 b \beta(1+e \cos \varphi)^{2}\left[2 \cos 2 \varphi-e \cos \varphi\left(1-3 \cos ^{2} \varphi\right)\right] \\
& F=-3 b \beta(1+e \cos \varphi)^{2}\left[2 \sin 2 \varphi+e \sin \varphi\left(1+3 \cos ^{2} \varphi\right)\right]
\end{aligned}
$$

where,

$$
\beta^{2}=\frac{4 G^{3} m_{1}{ }^{2} m_{2}^{2}\left(m_{1}+m_{2}\right)}{a^{5}\left(1-e^{2}\right)^{5}}
$$

Using formula (14) and averaging the resulting expressions over the orbital period, we obtain the intensity of $(\times)$ polarization component of gravitational wave from elliptical and oscillating binary, after a long calculation as

$$
\left\langle\frac{d I_{1}}{d \Omega}\right\rangle=\frac{G^{4} m_{1}^{2} m_{2}^{2}\left(m_{1}+m_{2}\right)}{\pi c^{5} a^{5}\left(1-e^{2}\right)^{7 / 2}}\left[\begin{array}{l}
\left(2+\frac{97}{16} e^{2}+\frac{49}{64} e^{4}\right) \cos ^{2} \theta+ \\
+b^{2}\left(2+\frac{111}{16} e^{2}+\frac{29}{32} e^{4}\right) \sin ^{2} \theta
\end{array}\right]
$$

This equation gives the average intensity of gravitational radiation per unit solid angle which depends on the oscillation parameter $b$ and eccentricity $e$. If $e=0$, i.e., for circular orbit, this equation reduces to equation (15). Note that $\omega$ in equation (15) is now given by $d \varphi / d t$ of equation (22) with $e=0$. The exactness of equations (15) and (26) for $e=0$ is clearly evident. For the $(+)$ polarization the calculations are more involved and lengthy. One would find the following result, (we have neglected terms of order higher than $b^{2}$ ):

$$
\begin{aligned}
& \left\langle\frac{d I_{2}}{d \Omega}\right\rangle=\frac{G^{4} m_{1}{ }^{2} m_{2}^{2}\left(m_{1}+m_{2}\right)}{\pi c^{5} a^{5}\left(1-e^{2}\right)^{7 / 2}} \times \\
& \times\left[\left(\frac{1}{2}+\frac{99}{64} e^{2}+\frac{51}{256} e^{4}-2 b^{2}-\frac{131}{16} e^{2} b^{2}-\frac{63}{64} e^{4} b^{2}\right) \cos ^{4} \theta\right. \\
& +\left(1+\frac{95}{32} e^{2}+\frac{47}{128} e^{4}+2 b^{2}+\frac{63}{8} e^{2} b^{2}+\frac{17}{16} e^{4} b^{2}\right) \cos ^{2} \theta \\
& \left.+\frac{1}{2}+\frac{99}{64} e^{2}+\frac{51}{256} e^{4}-\frac{15}{48} e^{2} b^{2}-\frac{5}{64} e^{4} b^{2}\right]
\end{aligned}
$$

For $e=0$, this equation correctly gives formula (18) for circular orbit (note that in (27) no $b^{4}$ term are included, however, in (18) there are). The results we have obtained here 
coincide with the results of Peters and Mathews [3] when we put $b=0$ in the formulae (26) and (27).

Next, we calculate the total energy radiated in GW in all directions per unit time as

$$
-\frac{d E}{d t}=I=\frac{32 G^{4} m_{1}^{2} m_{2}^{2}\left(m_{1}+m_{2}\right)}{5 c^{5} a^{5}\left(1-e^{2}\right)^{7 / 2}}\left[1+\frac{73}{24} e^{2}+\frac{37}{96} e^{4}+b^{2}\left(1+\frac{53}{16} e^{2}+\frac{41}{72} e^{4}\right)\right]
$$

which is generalization of the result of Landau and Lifshitz [4] for gravitational radiation from a Keplerian binary in elliptic orbit, now with a small oscillation of amplitude $b$. For numerical results, we note that this is known for binaries like PSR B 1913+16 without the oscillation induced term. The contribution from the oscillation can not be ascertained at present, because specific values of the parameter $b$ are not yet known. However, the presence of small oscillation is evident from theoretical investigation [14], this is why we have performed this study.

\section{Conclusion}

We have revisited an old problem, namely, energy radiated in gravitational wave by a Keplerian astrophysical binary system. In literature, we find this problem discussed in the context of a binary in circular or elliptical orbit with the plane of the orbit invariant [3-4]. Since, it is seen in literature [14] that due to spin-orbit interaction, the orbital plane in a physical binary may undergo oscillation in a manner whose one simplified form is a small oscillation about the equilibrium $\mathrm{x}-\mathrm{y}$ plane with the angular frequency of this oscillation as equal to the Newtonian orbital frequency, we found it as an interesting problem to find the quantity of GW radiation in such a case. Our problem is the canonical problem of GW radiation from a circular or elliptic binary, but with an additional parameter $b$ which is the amplitude of angular oscillation of the orbital plane (see, Eq. (9)). Our final result is depicted in Eqs. (20) and (28), which give the total energy radiated in all directions in GW per unit time averaged over the orbital period. The formulae we obtain, Eqs. (20) and (28), are an extension of the classic results of Landau and Lifshitz [4] for the case of GW radiation from circular or elliptic binary with fixed orbital plane. The oscillation we considered augments the energy of radiated GW by an amount which is simply dependent on the parameter $b$. Since our calculation is based on an approximation ( $\sin \theta \cong \theta$ ), our result predicts for such cases as applicable, a maximum increase of GW radiation energy by about $1 \%$ from the cases where there are no oscillation of the binary orbit. We consider our simple calculation presented here as a valuable addition to gravitational wave phenomenology that can arouse interest in practical GW researchers.

\section{Acknowledgement}

This research is supported by a financial grant from the Advanced Studies Board of Shahjalal University of Science and Technology, Sylhet, Bangladesh. 


\section{References}

1. D. Sigg, Class. Quantum Grav. 25, 114041 (2008). doi:10.1088/0264-9381/25/11/114041

2. D. A. Shaddock, Class. Quantum Grav. 25, 114012 (2008). doi:10.1088/0264-9381/25/11/114012

3. P. C. Peters and J. Mathews, Phys. Rev. 131, 435 (1963). doi:10.1103/PhysRev.131.435

4. L. D. Landau and E. M. Lifshitz, The Classical Theory of Fields, 4th Edition (Pergamon, Oxford, 1975).

5. H. Grote, Class. Quantum Grav. 25, 114043 (2008). doi:10.1088/0264-9381/25/11/114043

6. E. Berti, V. Cardoso, J. A. Gonzalez, U. Sperhake, and B. Brugmann, Class. Quantum Grav. 25, 114035 (2008). doi:10.1088/0264-9381/25/11/114035

7. G. Mendell and K. Wette, Class. Quantum Grav. 25, 114044 (2008). doi:10.1088/0264-9381/25/11/114044

8. D. Shoemaker, B. Vaishnav, I. Hinder, and F. Herrmann, Class. Quantum Grav. 25, 114047 (2008). doi:10.1088/0264-9381/25/11/114047

9. C. Cutler, D. Kennefick, and E. Poisson, Phys. Rev. D 50, 3816 (1994). doi:10.1103/PhysRevD.50.3816

10. F. D. Ryan, Phys. Rev. D 52, 3154 (1995). doi:10.1103/PhysRevD.52.5707

11. K. Glampedakis and D. Kennefick, Phys. Rev. D 66, 044002 (2002). doi:10.1103/PhysRevD.66.044002

12. L. Gergely and Z. Keresztes, Phys. Rev. D 67, 024020 (2003). doi:10.1103/PhysRevD.67.024020

13. A. Vecchio, Phys. Rev. D 70, 042001 (2004). doi:10.1103/PhysRevD.70.042001

14. B. Mashhoon and D. Singh, Phys. Rev. D 74, 124006 (2006). doi:10.1103/PhysRevD.74.124006

15. M. Zimmermann and E. Szedenits Jr., Phys. Rev. D 20, 351 (1999). doi:10.1103/PhysRevD.20.351

16. H. Beltrami and W.Y. Chau, Astrophys. Sp. Sci. 111, 335 (1985).

17. D. D. Dionysiou, Astrophys. Sp. Sci. 125, 115 (1986). doi:10.1007/BF00643977

18. M. Shibata, Phys. Rev. D 48, 663 (1993). doi:10.1103/PhysRevD.48.663

19. C. Moreno-Garrido, J. Buitrago, and E. Mediavilla, Mon. Not. R. Astron. Soc. 266, 16 (1994).

20. C. Moreno-Garrido, J. Buitrago, and E. Mediavilla, Mon. Not. R. Astron. Soc. 274, 115 (1995).

21. L. Blanchet, Phys. Rev. D 54, 1417 (1996). doi:10.1103/PhysRevD.54.1417

22. C. S. Kochanek, S. L. Shapiro, S. A. Teukolsky, and D. F. Chernoff, Astrophys. J. 358, 81 (1990). doi:10.1086/168964 